\title{
$R$ measurements with ISR in BaBar - hadronic part of muon magnetic dipole moment
}

\author{
Paul Taras \\ Université de Montréal, Physique des Particules, Montréal, Québec, Canada H3C 3J7 \\ representing the BaBar Collaboration
}

\begin{abstract}
Recent measurements of the quantity $R$, the ratio of annihilation $\sigma$, including those following Initial State Radiation, are discussed in the context of the hadronic part of $\mu$, the muon magnetic dipole moment. The data indicate that more precise theoretical and experimental values of $\mu$ are needed to establish whether new physics has been observed in the measurement of $\mu$.
\end{abstract}

Keywords: annihilation cross sections, ISR, muon magnetic moment

PACS: $13.66 . \mathrm{Bc}, 13.25 . \mathrm{Gv}, 13.25 . \mathrm{Jx}, 14.40 . \mathrm{Cs}, 14.60 . \mathrm{Ef}$

\section{INTRODUCTION}

The recent [1] very precise measurement of the muon g-factor hinted at a possibility of new physics because of a small discrepancy with its value expected in the framework of the Standard Model. This presentation will dwell on the extraction of the leading order part of the hadronic component of the muon magnetic dipole moment from the various available values of $R$, the ratio of annihilation cross sections.

\section{MEASUREMENTS OF R AND THEIR IMPACT ON THE MUON MAGNETIC DIPOLE MOMENT PROBLEM}

\section{The magnetic anomaly in the framework of the Standard Model}

It is well known that the magnetic dipole moment of a spin $\frac{1}{2}$ particle is given by: $\vec{\mu}=g \frac{e}{2 m} \vec{s}$ where in the case of the muon, $e, m$ and $\vec{s}$ are the charge, the mass and the spin of the muon while, in quantum mechanics, the gyromagnetic factor $g \equiv 2$ for all spin $\frac{1}{2}$ particles. However, soon after quantum electrodynamics was developped, it was realized that the value of $g$ needed to be slightly modified. This was written as $g=2+2 a$, where by definition, $a \equiv(g-2) / 2$ is known as the magnetic anomaly. In the framework of the Standard model, the value of $a$ has three components :

$$
a_{\mu}^{S M}=a_{\mu}^{Q E D}+a_{\mu}^{E W}+a_{\mu}^{h a d}
$$

The values of $a_{\mu}^{Q E D}$, the QED component [2] and $a_{\mu}^{E W}$, the electroweak processes'component [3] are calculated with high precision, to 5 and 2 loops, respectively, and need not concern us here. The hadronic component, $a_{\mu}^{\text {had }}$ can be broken down into 
three parts :

$$
a_{\mu}^{h a d}=a_{\mu}^{h a d, L o}+a_{\mu}^{h a d, H o}+a_{\mu}^{h a d, L B L}
$$

$a_{\mu}^{\text {had }, L o}$, the leading order part of the hadronic interaction arising from the contribution of the hadronic vacuum polarization [4], $a_{\mu}^{\text {had,Ho }}$, the three-loop part of the hadronic interaction involving one hadronic vacuum polarization insertion [4], and $a_{\mu}^{\text {had,LBL }}$, the so-called hadronic "light-by-light" scaterring part [5, 6, 4].

\section{The relation between $R$ and the magnetic anomaly}

The contribution of the hadronic vacuum polarization is calculated via the dispersion integral $[4,7]$ :

$$
a_{\mu}^{\text {had }, L o}=\left(\frac{\alpha m_{\mu}}{3 \pi}\right)^{2} \int_{4 m_{\pi}^{2}}^{\infty} \frac{K(s)}{s^{2}} R(s) d s
$$

where :

$$
R(s)=\frac{\sigma_{f}\left(e^{+} e^{-} \rightarrow \text { hadrons }\right)}{\sigma_{f}\left(e^{+} e^{-} \rightarrow \mu^{+} \mu^{-}\right)}
$$

is the ratio of the direct annihilation cross section, $\sigma_{f}$, to a given hadronic final state $f$ to that of the muon pair production. This ratio $R(s)$ is measured as a function of $\sqrt{s}$, the center of mass energy of the final system $f$. The QED kernel, $K(s)$ varies very little over the whole range [7], from 0.63 at $s=4 m_{\pi}^{2}$ to 1 at $s=\infty$. There is, however, a very strong dependence on energy. Because the main theoretical error in $a_{\mu}^{S M}$ arises from the uncertainties in $a_{\mu}^{\text {had }}$, it is of the utmost importance to measure the values of $R(s)$ as precisely as possible, specially at lower energies since about $91 \%$ of the total contribution to $a_{\mu}^{\text {had,Lo }}$ is provided by the cross sections measured at energies $\sqrt{s} \leq 1.8$ $\mathrm{GeV}$. Monte Carlo calculations show that many channels open up as the energy available for the final state increases : $\pi^{+} \pi^{-} \pi^{0}, \pi^{+} \pi^{-} \pi^{0} \pi^{0}, K^{+} K^{-} \pi^{+} \pi^{-}$, etc ... but the most important final state is $\pi^{+} \pi^{-}$which contributes up to $73 \%$ of the total value of $a_{\mu}^{\text {had, Lo }}$.

\section{Annihilation cross sections}

The best existing $e^{+} e^{-} \rightarrow \pi^{+} \pi^{-}$data are provided by the CMD-2, SND and KLOE collaborations. Although they can be combined to provide a precise contribution of $(376.5 \pm 0.8 \pm 2.4) \times 10^{-10}$ to the value of $a_{\mu}^{\text {had,Lo }}$, there is some discrepancy between the KLOE data set and the others which needs to be resolved. In addition, the pion form factor extracted from the annihilation cross sections using the conserved vector current hypothesis is not in agreement [4] with that obtained from the branching fraction $B F\left(\tau^{-} \rightarrow \pi^{-} \pi^{0} v_{\tau}\right)$, once all sources of isospin symmetry breaking have been taken into account. This may be due to the use of incorrect spectral functions and merits further investigation. In any case, it is clear that an independent measurement of this very important cross section is required. 
TABLE 1. Samples of contributions to $a_{\mu}^{\text {had,Lo }}$ including BaBar data following ISR

\begin{tabular}{crrr}
\hline $\begin{array}{c}\text { Integrated } \\
\text { luminosity } f b^{-1}\end{array}$ & Final state & $\begin{array}{r}a_{\mu}^{\text {had,Lo }} \times 10^{10} \\
\text { without BaBar data }\end{array}$ & $\begin{array}{r}a_{\mu}^{\text {had,Lo }} \times 10^{10} \\
\text { with BaBar data }\end{array}$ \\
\hline 89.3 & $\pi^{+} \pi^{-} \pi^{0}$ & $2.45 \pm 0.26 \pm 0.03$ & $3.25 \pm 0.09 \pm 0.01$ \\
89.3 & $2 \pi^{+} 2 \pi^{-}$ & $14.20 \pm 0.87 \pm 0.24$ & $13.09 \pm 0.44 \pm 0.00$ \\
232 & $3 \pi^{+} 3 \pi^{-}$ & $0.10 \pm 0.10$ & $0.108 \pm 0.016$ \\
232 & $2 \pi^{+} 2 \pi^{-} 2 \pi^{0}$ & $1.42 \pm 0.30 \pm 0.03$ & $0.890 \pm 0.093$ \\
\hline
\end{tabular}

In $B a B a r$, the annihilation cross sections for the final states of interest are measured following Initial State Radiation. Depending on the energy of the radiated photon, the cross section for the reconstructed final state can be obtained from threshold to nominally $\sqrt{s}$, the c.m. energy of the PEP-II collider in a single experiment at $\sqrt{s}$. This program is made possible by the high luminosity of our collider and the excellent BaBar detector. The statistics obtained are comparable to those of CMD-2 and SND at $E_{c m} \leq 1.4 \mathrm{GeV}$, and much better than those of DM1 and DM2 at higher energies. The measurements following ISR contain little background and should thus lead to high accuracy even for most of the exclusive decays which have a rather low cross section.

Only a portion of the available data is presented here to illustrate the precision achieved with ISR in the BaBar experiment. These are given in Table 1 for the $e^{+} e^{-} \rightarrow \pi^{+} \pi^{-} \pi^{0}$ [9], $e^{+} e^{-} \rightarrow 2 \pi^{+} 2 \pi^{-}$[10], $e^{+} e^{-} \rightarrow 3 \pi^{+} 3 \pi^{-}$[11] and $e^{+} e^{-} \rightarrow 2 \pi^{+} 2 \pi^{-} 2 \pi^{0}$ [11] channels. In the table, we list the contributions to the leading order hadronic part, $a_{\mu}^{h a d, L o}$, first without taking into account the BaBar data, and then doing so. The considerable gain in precision in doing so (factor of 2 to 5) results from the fact that the present BaBar data is more accurate and cover a larger energy range than previous data for these same final states. We should note that the large improvement in accuracy in the $\pi^{+} \pi^{-} \pi^{0}$ channel is due in part to the removal of the DM2 data shown to be incorrect by our data.

\section{DISCUSSION AND CONCLUSIONS}

The precisely measured [1] value of the magnetic anomaly : $\left.a_{\mu}^{\exp }=11659208.0 \pm 6.3\right) \times$ $10^{-10}$ can be compared to the value computed in the framework of the standard model, $\left.a_{\mu}^{S M}=11659180.5 \pm 5.6_{\text {had }} \pm 0.2_{Q E D+E W}\right) \times 10^{-10}$ using all known annihilation cross section data, as reported by Eidelman at ICHEP06 [7]. This value includes the three hadronic contributions : $a_{\mu}^{\text {had,Lo }}=(690.9 \pm 3.9 \pm 2.0) \times 10^{-10}[7], a_{\mu}^{\text {had, }{ }^{H o}}=-(9.8 \pm$ $0.1) \times 10^{-10}[4]$ and $a_{\mu}^{\text {had, } L B L}=+(12.0 \pm 3.5) \times 10^{-10}[4,5,6]$. The last two terms are rather small but still significant in light of the high precision achieved experimentally. Comparing the values, it appears that there is a difference of $3.3 \sigma$ between the measured and the phenomenological values. Is this a sign of New Physics?

Let us consider the results shown in Fig. 1 where the band represents the experimental value while the full lines show the values obtained from the annihilation cross sections and the dashed line that from the $\tau$ decay branching fraction [4]. It is clear that although 


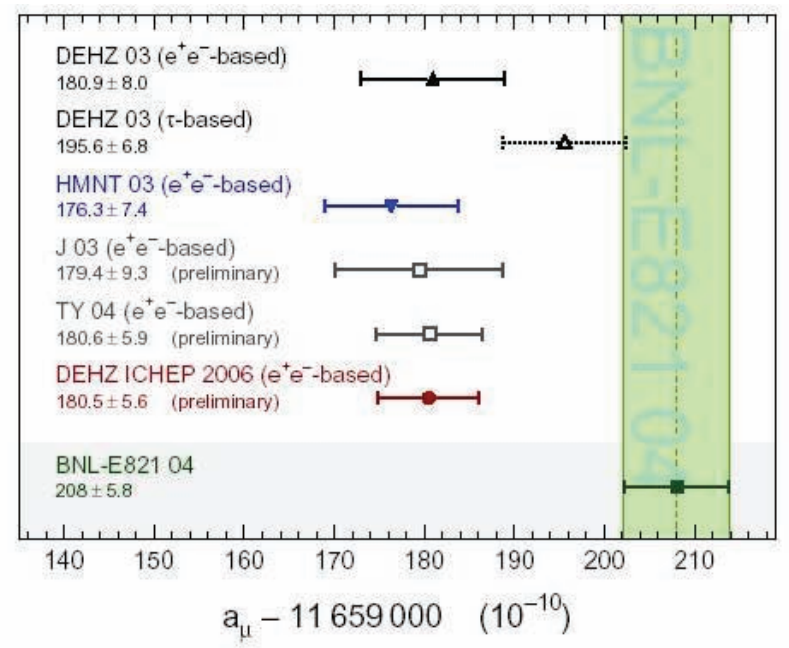

FIGURE 1. Experimental and SM values of the magnetic anomaly

there is a significant difference between the experimental value and the one based on the cross sections, the value based on the branching fraction is compatible with the measurement. In addition, as reported [12] at ICHEP06, recent lattice QCD calculations could also be compatible with the measurement. Furthermore, my own logic dictates that the theoretical errors generated in the computation of $a_{\mu}^{\text {had }}$ must be increased. The same point was made by the summary conference speaker [13] at ICHEP06. Thus, more precise theoretical and experimental values of $\mu$ are needed to establish whether new physics has indeed been observed in the measurement of the muon magnetic moment.

\section{ACKNOWLEDGMENTS}

M. Davier, S. Eidelman and E. Solodov are thanked for many fruitful discussions. This work is partially supported by NSERC (Canada).

\section{REFERENCES}

1. G.W. Bennett et al., Phys. Rev. D73, 072003 (2006).

2. G. Gabrielse et al., Phys. Rev. Lett. 97, 030802 (2006).

3. A. Czarnecki, W. Marciano, A. Vainshtein, Phys. Rev. D67, 073006 (2003).

4. M. Davier, W. Marciano, Annu. Rev. Nucl. Part. Sci. 54, 115 (2004).

5. M. Knecht, A. Nyffeler, Phys. Rev. Lett. 88, 071802 (2002).

6. K. Melnikov, A. Vainshtein, hep-ph/0312226.

7. S. Eidelman, International Conference on High Energy Physics, Moscow, 2006, contributed paper.

8. R. Akhmetsin et al., CMD-2 collaboration, hep-ex/0308008

9. B. Aubert et al. (BaBar collaboration), Phys. Rev. D70, 072004 (2004).

10. B. Aubert et al. (BaBar collaboration), Phys. Rev. D71, 052001 (2005).

11. B. Aubert et al. (BaBar collaboration), Phys. Rev. D73, 052003 (2006).

12. G. Schierholz, International Conference on High Energy Physics, Moscow, 2006, invited paper.

13. V. A. Rubakov, International Conference on High Energy Physics, Moscow, 2006, summary paper. 